\title{
Evaluation of inflamed coronary atherosclerotic plaques by PET: More evidences for a promising area of cardiovascular imaging
}

\section{To the Editor,}

In the latest years, there is a growing interest in the early detection of inflamed coronary atherosclerotic plaques as major determinants of the risk of developing a symptomatic coronary artery disease (CAD). The possibility of using non-invasive techniques to identify high-risk lesions would be highly desirable both for prevention and for treatment evaluation of CAD.

18F-FDG PET/CT is a widespread technique able to detect inflammatory foci but, unfortunately, it is well known that the unpredictable myocardial glucose metabolism can be a serious limitation in its clinical use.

A recent paper by Joshi et al $^{1}$ evaluated 18F-fluoride $\mathrm{PET} / \mathrm{CT}$ in the assessment of atherosclerotic plaques; despite limitations concerning some missing clinical information and the lack of images with a quantitative scale, the study seemed to indicate that $18 \mathrm{~F}$ fluoride is a better tracer to evaluate the presence of active calcification, macrophage infiltration, apoptosis, and necrosis as seen in culprit lesions. On the contrary, $18 \mathrm{~F}-\mathrm{FDG}$ was not considered to be sufficiently robust for a clinical application to the coronary circulation. ${ }^{1}$

Other possible tracers of inflammation such as somatostatin-analogs have been also studied. A paper by Schatka et $\mathrm{al}^{2}$ reported that $68 \mathrm{Ga}$-DOTATATE PET/CT can characterize biologic activity of atherosclerotic plaque via SSTR-2 expression and that a targeted therapy with DOTATATE-based SSTR-2 results in a reduction of atherosclerotic plaque activity, thus suggesting a possible therapy strategy.

Now, the work by Blomberg et $\mathrm{al}^{3}$ adds another tessera in the process of optimizing PET/CT protocols in the evaluation of CAD and seems to rehabilitate FDG as an important tracer of atherosclerotic lesions.
In fact, the authors demonstrated that delayed 18F-FDG PET/CT imaging improves quantitation of atherosclerotic plaque inflammation over early imaging. Although the results are obtained evaluating large vessels, the same principle could be also transferred to coronary artery imaging. A strict dietary preparation ${ }^{4}$ along with a delayed scan could represent an important improvement in PET protocols, able to grant a successful and widespread clinical application to 18F-FDG.

\author{
Federico Caobelli, MD, FEBNM \\ Department of Nuclear Medicine, \\ Fondazione Poliambulanza. \\ Via Bissolati 57, 25100 \\ Brescia, Italy \\ fedefournier@libero.it
}

\section{References}

1. Joshi NV, Vesey AT, Williams MC, Shah AS, Calvert PA, Craighead $\mathrm{FH}$, et al. 18F-fluoride positron emission tomography for identification of ruptured and high-risk coronary atherosclerotic plaques: A prospective clinical trial. Lancet. 2014;383:705-13.

2. Schatka I, Wollenweber T, Haense C, Brunz F, Gratz KF, Bengel FM. Peptide receptor-targeted radionuclide therapy alters inflammation in atherosclerotic plaques. J Am Coll Cardiol. 2013;62:2344-5.

3. Blomberg BA, Thomassen A, Takx RA, Hildebrandt MG, Simonsen JA, Buch-Olsen KM, et al. Delayed 18F-fluorodeoxyglucose PET/CT imaging improves quantitation of atherosclerotic plaque inflammation: Results from the CAMONA study. J Nucl Cardiol. doi:10.1007/s12350-014-9884-6.

4. Williams G, Kolodny GM. Suppression of myocardial 18F-FDG uptake by preparing patients with a high-fat, low-carbohydrate diet. AJR Am J Roentgenol. 2008;190:W151-6.

doi:10.1007/s12350-014-9909-1 